\title{
Identification and characterization of an operon, $m s a A B C R$, that controls virulence and biofilm development in Staphylococcus aureus
}

Gyan S Sahukhal ${ }^{*}$ and Mohamed O Elasri

\begin{abstract}
Background: Community-acquired, methicillin-resistant Staphylococcus aureus strains often cause localized infections in immunocompromised hosts, but some strains show enhanced virulence leading to severe infections even among healthy individuals with no predisposing risk factors. The genetic basis for this enhanced virulence has yet to be determined. $S$. aureus possesses a wide variety of virulence factors, the expression of which is carefully coordinated by a variety of regulators. Several virulence regulators have been well characterized, but others have yet to be thoroughly investigated. Previously, we identified the msa gene as a regulator of several virulence genes, biofilm development, and antibiotic resistance. We also found evidence of the involvement of upstream genes in msa function.

Results: To investigate the mechanism of regulation of the msa gene (renamed msaC), we examined the upstream genes whose expression was affected by its deletion. We showed that msaC is part of a newly defined four-gene operon ( $m s a A B C R$ ), in which msaC is a non-protein-coding RNA that is essential for the function of the operon. Furthermore, we found that an antisense RNA ( $m s a R$ ) is complementary to the $5^{\prime}$ end of the msaB gene and is expressed in a growth phase-dependent manner suggesting that it is involved in regulation of the operon.

Conclusion: These findings allow us to define a new operon that regulates fundamental phenotypes in S. aureus such as biofilm development and virulence. Characterization of the msaABCR operon will allow us to investigate the mechanism of function of this operon and the role of the individual genes in regulation and interaction with its targets. This study identifies a new element in the complex regulatory circuits in S. aureus, and our findings may be therapeutically relevant.
\end{abstract}

Keywords: Staphylococcus aureus, msaABCR operon, Biofilm, Virulence factor

\section{Background}

Staphylococcus aureus is an important human pathogen that causes a wide range of infections, from superficial to systemic $[1,2]$. The ability of $S$. aureus to infect a variety of tissues is due to its expression of a wide variety of virulence factors. These factors are categorized as surface-associated proteins, secreted proteases, toxins, or immune modulators [3]. Expression of virulence factors in $S$. aureus is carefully coordinated by a variety of regulators that include trans-acting global regulators, alternative sigma factors, and small non-coding RNAs [3-5]. Indeed, the $S$. aureus genome has 124 putative transcriptional regulators [6]. Understanding virulence

\footnotetext{
* Correspondence: gyan.sahukhal@eagles.usm.edu; mohamed.elasri@usm.edu Department of Biological Sciences, The University of Southern Mississippi, Hattiesburg, Mississippi 39406-0001, USA
}

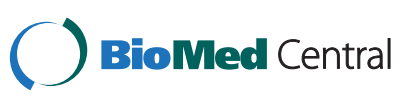

(c) 2014 Sahukhal and Elasri; licensee BioMed Central Ltd. This is an Open Access article distributed under the terms of the Creative Commons Attribution License (http://creativecommons.org/licenses/by/2.0), which permits unrestricted use, distribution, and reproduction in any medium, provided the original work is properly credited. The Creative Commons Public Domain Dedication waiver (http://creativecommons.org/publicdomain/zero/1.0/) applies to the data made available in this article, unless otherwise stated. regulation during growth under different environmental conditions (e.g., biofilm development) is imperative for the effective prevention and treatment of $S$. aureus infections. To date, several global regulators have been identified, which include the $a g r$ operon, the $s a r A$ gene family, the saePQRS operon, and the genes arlRS, lytSR, srrAb, hssRS, vraSR, and graSR [7-16]. Several other regulators have also been identified, though they are not as well characterized (e.g., htrA, $c c p A, m s r R$, and $s v r R$ ) [17-20].

The msa gene, henceforth referred to as $m s a C$, was initially identified as a regulator of sarA, agr, and several virulence factors [21]. We previously reported that $m s a C$ is also involved in biofilm development [22]. Indeed, we showed that deletion of the $m s a C$ gene resulted in a significant defect in accumulation of biofilm but did not affect the initial adherence to surfaces [22]. However, it was not clear if $m s a C$ regulated virulence genes directly 
or via its effect on global regulators. For instance, we found that in the msaC deletion mutant, sarA expression was reduced during biofilm growth. Since sarA has been shown to be essential for biofilm development in several strains [23], it is not clear if the msaC defect is due to the reduction in sarA or other factors. Sequence analysis of the $m s a C$ gene showed that it is conserved among $S$. aureus strains and suggested that it encodes a putative membrane protein [21]. We have been unable to express this putative protein, and therefore the mechanism of regulation by $m s a C$ remains to be determined. The pleiotropic phenotypes in the $m s a C$ mutant suggested that they were mediated by the global regulators sarA, $a g r$, and $\operatorname{sig} B$. However, deletion of the $m s a C$ gene leads to a decrease in the expression of the upstream gene $(\operatorname{csp} A)$, a gene that is not regulated by $\operatorname{sar} A, \operatorname{agr}$, or $\operatorname{sig} B$ $[21,24]$. These findings led us to hypothesize that $m s a C$ regulated some genes (e.g., cspA) directly. Here, we examined the relationship between $m s a C$ and $\operatorname{csp} A$ and showed that the $m s a C$ gene is part of a four-gene operon.

\section{Results}

$m s a C$ is a member of a four-gene operon

Previously, we showed that $m s a C$ regulates the expression of sarA, and is essential for biofilm formation and protease production $[21,22]$. One of the early observations about $m s a C$ was that expression of the upstream gene (SAUSA300_1295) was significantly reduced (twofold) by deletion of $m s a C$ [21]. In addition, some assays involving expression of upstream genes did not show full complementation when we introduced the msaC gene alone into the msaC mutant. To investigate this further, we deleted the $m s a C$ gene from the $S$. aureus community-acquired strain, USA300 LAC, and complemented the mutant with a region of the chromosome that included upstream and downstream genes relative to $m s a C$. Introduction of three open reading frames (ORFs) (encoding SAUSA300_1296, SAUSA300_1295, and $m s a C$ ) resulted in full complementation to restore the wild type phenotype in biofilm formation, host protein binding assays, protease production, and expression of $s a r A$. These findings suggested that the $m s a C$ gene is part of an operon that included at least three genes. To test this hypothesis, we deleted all three genes (encoding SAUSA300_1296, encoding SAUSA300_1295, and msaC) in the USA300 LAC strain and compared the phenotype to the $m s a C$ single-deletion mutant. Several phenotypic variables were examined in the two mutants and similar phenotypes were observed. Both mutants showed reduced pigmentation and biofilm production and increased protease activity relative to wild type (Figure 1). Both mutants also showed a downregulation in the expression of key global regulators, sarA, agrA, and $\operatorname{sig} B$ (Table 1). The phenotype of the three-ORF-deletion mutant was similar to the $m s a C$ mutant, with one exception being that the three-ORF-deletion mutant produced significantly more proteases than the $m s a C$ mutant (Figure 1B). Furthermore, complementation studies in the $m s a C$ deletion mutant confirmed that all three ORFs were required for restoration of the wild type phenotype (Figure 1, Table 1). These findings suggested that the three genes are functionally related and form an operon.

These findings led us to analyze the transcript produced by these three ORFs using rapid amplification of cDNA ends (RACE) and Northern blot analyses. We used the SMARTer ${ }^{\mathrm{Tm}}$ RACE cDNA Amplification kit (Clontech) to analyze the mRNA transcripts harvested from the USA300 LAC strain. We used several genespecific primers to find the $5^{\prime}$ and $3^{\prime}$ ends of all transcripts produced by this operon. The RACE reaction products were separated by agarose gel electrophoresis, and the different DNA bands observed were gel purified and sequenced. The results from RACE analysis $\left(5^{\prime}\right.$ and $\left.3^{\prime}\right)$ showed the production of a 1541-nt RNA whose 5' end was located $35 \mathrm{nt}$ upstream of the gene encoding SAUSA300_1296 (renamed $m s a A$ ), while its 3' end was located $230 \mathrm{nt}$ downstream of the gene encoding SAUSA300_1294 (msaC). The ends of each transcript were confirmed using at least two different primers and three independent reactions for each primer (Figure 2). Results of the RACE analysis were confirmed by Northern blot analysis using gene-specific probes (Figure 3).

The findings from RACE and Northern analyses confirmed that $m s a C$ is part of a three-gene operon that comprises a gene that encodes for a hypothetical protein (SAUSA300_1296), a gene that is similar to the E. coli cold-shock gene (cspA, encoding SAUSA300_1295), and the $m s a C$ gene (encoding SAUSA300_1294) (Figures 2 and 3). This indicated that all three genes were functionally related and involved in biofilm development, protease production, and regulation of the $\operatorname{sar} A$, agr, and $\operatorname{sig} B$ genes. We named this operon $m s a A B C R$, where $m s a A$ encodes SAUSA300_1296, msaB encodes SAUSA300_1295, $m s a C$ encodes SAUSA_1294 (originally named $m s a$ ), and $m s a R$ codes for an anti-sense RNA (see below).

Another interesting finding from RACE and Northern blot analyses was that in addition to the large transcript, there were three sub-transcripts corresponding to $m s a A B$, $m s a B$, and $m s a C$ (Figures 2 and 3). Additionally, Northern blot analysis using an $m s a A$-specific riboprobe showed the presence of a transcript that corresponded to $m s a A$ alone, however, we were not able to confirm the ends of this transcript by RACE (Figure 3). Northern blot analysis also revealed that $m s a B$ was the most abundant transcript produced from this operon, whereas the large msaABC transcript and the $m s a C$ transcript were present at a much lower level (Figure 3). The expression level of these 


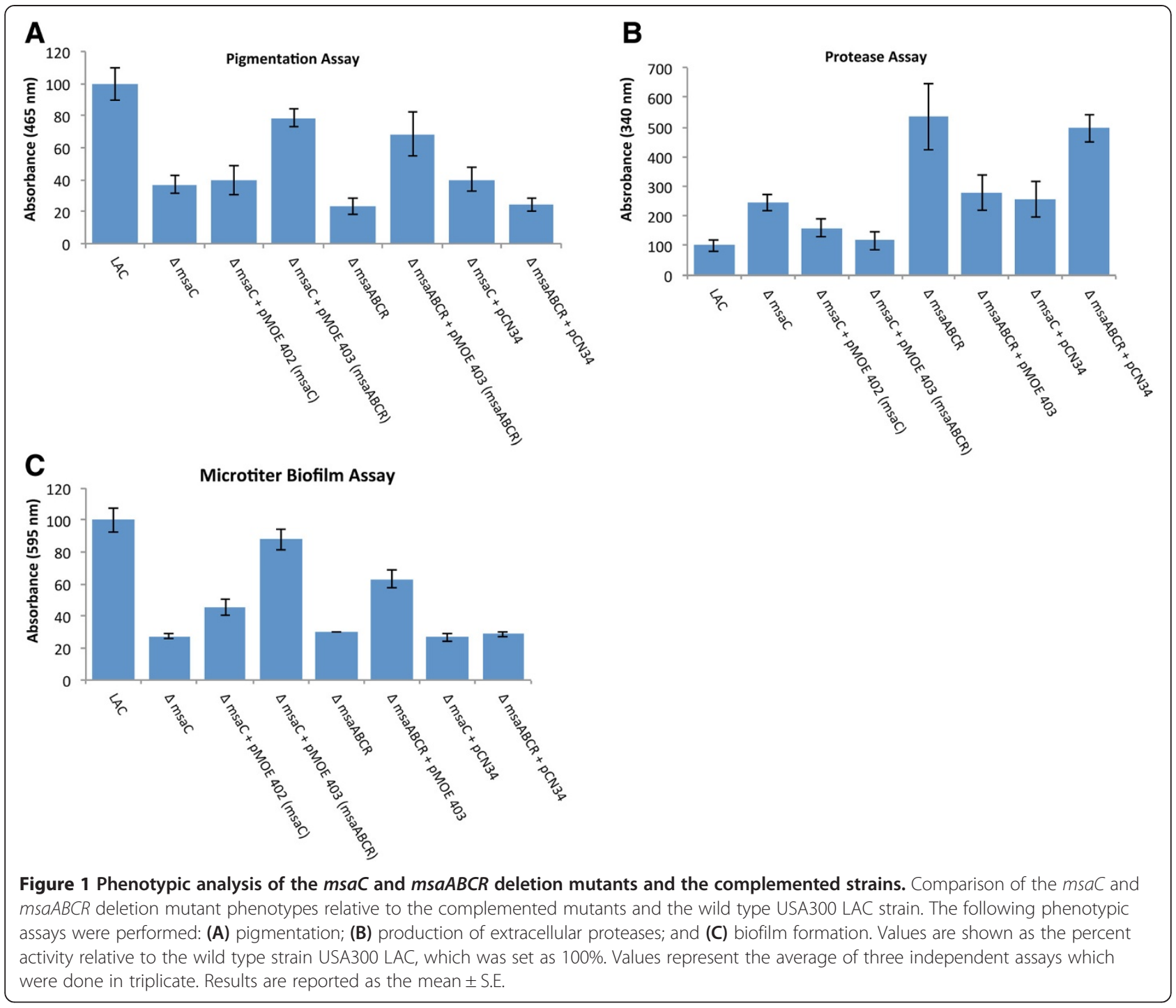

Table 1 Relative expression of global regulators (sarA, $a g r, s i g B$ ) in the $m s a C$ and $m s a A B C R$ deletion mutants

\begin{tabular}{llll}
\hline Strain & \multicolumn{3}{l}{ Fold change* $^{*}$} \\
\cline { 2 - 4 } & sarA & agrA & sigB \\
\hline $\mathrm{LAC}$ & 1 & 1 & 1 \\
$\triangle m s a C$ & -3.03 & -5.72 & -2.37 \\
$\triangle m s a C+$ pMOE402 (msaC) & -3.45 & -6.74 & -2.23 \\
$\triangle m s a C+$ pMOE403 (msaABCR) & -1.74 & 1.74 & -1.35 \\
$\triangle m s a A B C R$ & -3.59 & -6.7 & -2.07 \\
$\triangle m s a A B C R+$ pMOE403 (msaABCR) & -1.39 & -1.56 & 1.01 \\
\hline
\end{tabular}

*Fold change in the transcription level in the various strains relative to wild type (USA300 LAC). Values represent the mean ratio for at least three independent experiments. transcripts was further confirmed by real-time quantitative PCR (RT-qPCR) (Additional file 1: Figure S1). These results suggested that the large transcript undergoes posttranscriptional processing to produce the final $m s a B$ transcript. However, the mechanism of production of the abundant $m s a B$ transcript requires further studies.

\section{The $m s a A B C R$ operon contains an anti-sense RNA ( $m s a R$ )}

We used a sense riboprobe that hybridizes to the $m s a B$ region and performed Northern blot analysis. The probe detected an anti-sense RNA that partially overlaps with the $m s a B$ transcript. We performed RACE analysis to identify the ends of the anti-sense RNA and found that it is $133 \mathrm{nt}$ in length and is complementary to $112 \mathrm{nt}$ of the $5^{\prime}$ UTR region of $m s a B$ and $18 \mathrm{nt}$ of the $m s a B$ ORF region (Figure 2). Our results are supported by the identification of this antisense RNA in a screen of endoribonuclease III targets in the S. aureus strain RN6390 [25]. Lioliou et al. [25] found 


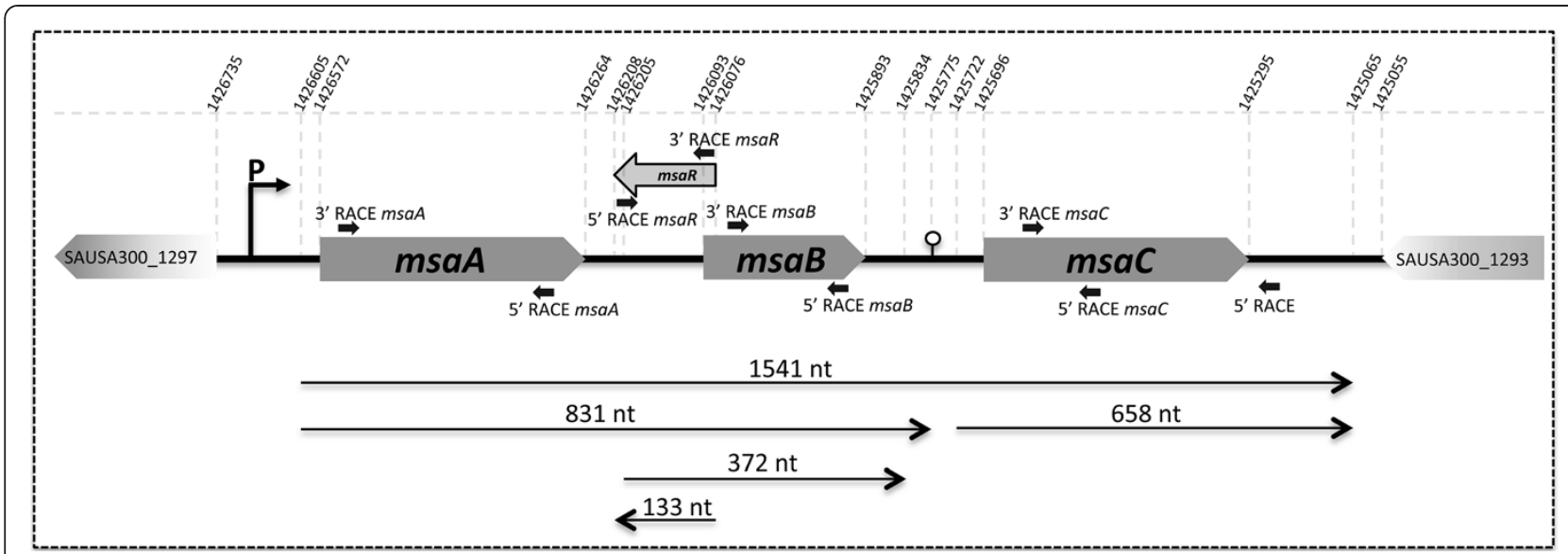

Figure 2 Map of the msaABCR operon. Rapid amplification of cDNA ends (RACE) and Northern blot analyses were used to determine the length and ends ( $5^{\prime}$ and $3^{\prime}$ ) of the RNA transcripts produced by the msaABCR operon. Long thin arrows represent the various RNAs identified in this study, the direction and length in nucleotides ( $\mathrm{nt}$ ) is also indicated. Short thick arrows indicate the positions and directions of the gene-specific primers used in the separate reactions. Reporter gene fusion studies showed the presence of only one active promoter (P) in this operon.

that this 133-nt RNA is involved in regulating the stability of the $\operatorname{csp} A$ (msaB) mRNA. To investigate this further, we measured the expression level of this anti-sense transcript at three growth phases and found that it was produced at early-exponential and mid-exponential phases but it was

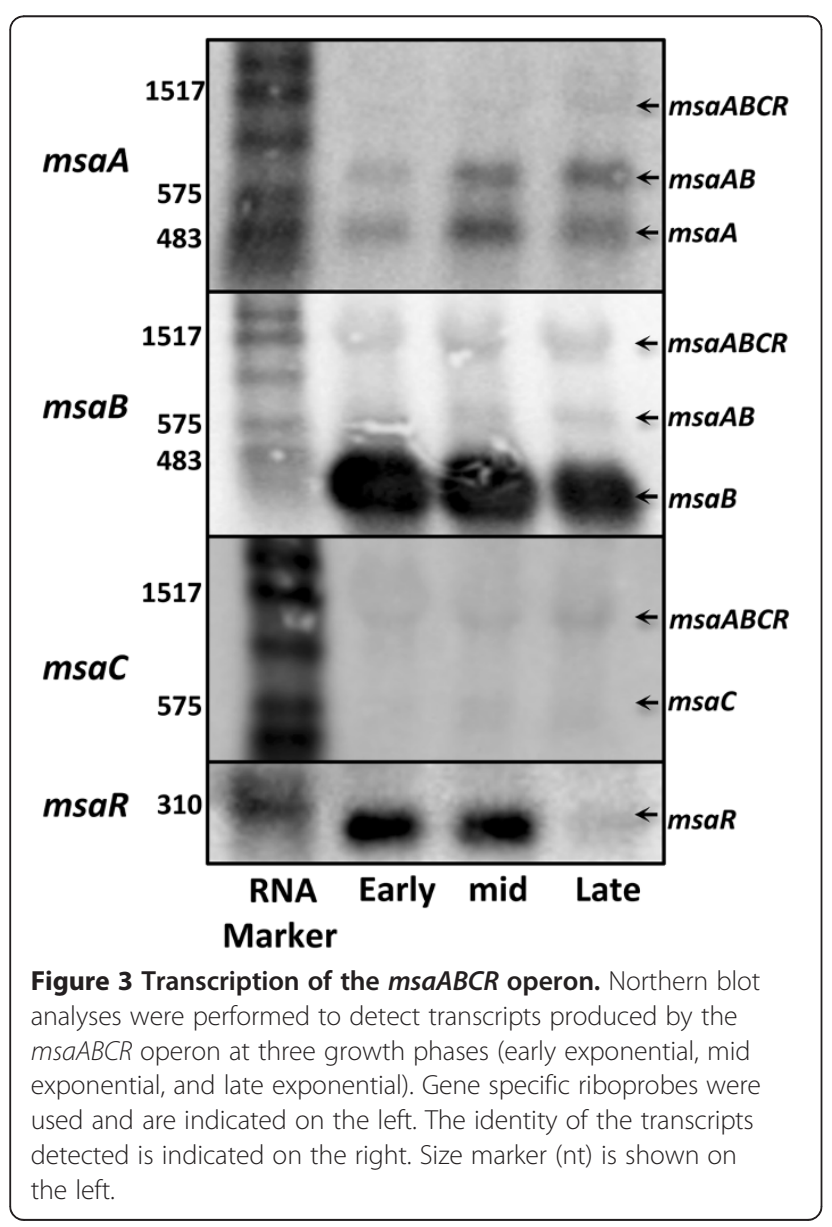

absent at late-exponential phase (Figure 3). The absence of the anti-sense transcript in the late-exponential growth phase may be due to lack of production or degradation. This differential expression may play an important role in the activity of the operon and suggests that regulation of the operon by msaR might be growth-phase dependent.

\section{Detectable promoter activity within the operon is limited} to $m s a A$

RACE and Northern blot analyses showed that, in addition to the large transcript ( $m s a A B C$ ), the operon produces four sub-transcripts ( $m s a A B, m s a B, m s a C$, and $m s a R$ ). These transcripts might be produced from individual promoters or via processing of the large transcript. To test this, we fused the putative promoters from all of the genes in the operon to a promoterless luciferase gene, $\operatorname{lux} A B$. We used two promoter-prediction software packages, PePPER (prediction of prokaryote promoter elements and regulon) [26] and Virtual footprint (promoter analysis version 3.0) [27], to select the putative promoters. The promoters included 250 to $300 \mathrm{bp}$ of sequence upstream of each ORF. Selection of the putative promoter regions was also based on the results of RACE analysis by considering every detected $5^{\prime}$ end as a possible transcription start point. We introduced the fusions into the wild type strain and assayed for light production in three growth phases (early-exponential, mid-exponential, and late-exponential). We found that under these growth conditions, the $m s a A$ promoter was active in all three-growth phases (Figure 4). However, the putative promoters for $m s a B$ and $m s a C$ did not show any detectable light production (Figure 4). Compared to controls, the $m s a A$ promoter shows higher activity than the serine protease promoter but lower than the sarAP1 promoter (Figure 4). These findings further confirmed that a 


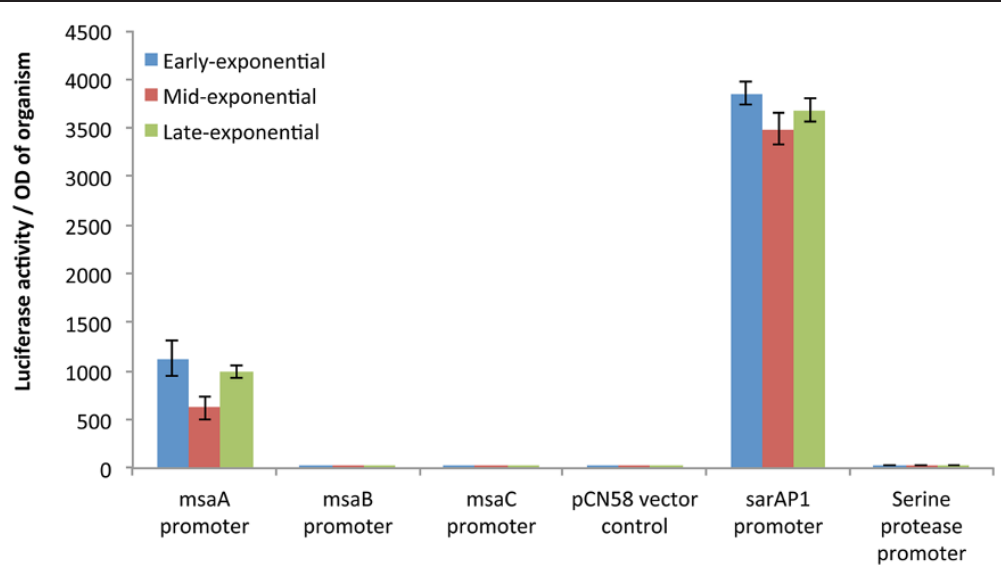

Figure 4 Promoter activity of $m s a A, m s a B$, and $m s a C$ at three growth phases. Putative promoters were fused to promoter-less /uxAB genes and introduced into the wild type strain USA300 LAC. Luciferase activity was measured at three growth phases (early, mid and late exponential). The vector pCN58 containing luXAB without a promoter was used as a negative control. sarAP1 and serine protease promoters were used as positive controls. Results represent means of three independent experiments where each measurement was done in triplicates. Standard error bars are shown.

single promoter drives expression of the genes in the $m s a A B C R$ operon and that the sub-transcripts probably arise from processing of the main transcript. However, we cannot completely rule out the function of the other putative promoters, which may only be active under growth conditions that we have not yet tested.

To further examine the potential role of the $m s a A$ promoter in the regulation of expression of the operon, we introduced msaA-luxAB fusions into the two deletion mutants, the $m s a A B C R$ operon, and $m s a C$. We found that the promoter activity was significantly increased ( $>2$ fold) in both mutants, indicating a negative auto-regulation mechanism that controls the expression of the operon (Additional file 2: Figure S2). Interestingly, this increase in expression was observed in three phases of planktonic growth as well as in biofilms.

\section{msaC is a non-protein-coding RNA}

Studies on the $m s a C$ gene were initiated by the discovery of its role in the expression of sarA and several virulence factors. In this study, we showed that $m s a C$ is part of a four-gene operon, $m s a A B C R$, and that its deletion leads to a phenotype that is equivalent to deletion of the whole operon. Lack of similarity of the predicted protein sequence encoded by $m s a C$ with protein databases and our failure to express the protein using several approaches led us to hypothesize that $m s a C$ is a non-coding RNA. We constructed a mutant in the $m s a A B C R$ operon that contained a frameshift mutation in the $m s a C$ ORF that did not significantly change the predicted secondary structure of the RNA (Additional file 3: Figure S3.A). This frame shift mutation led to the introduction of several nonsense mutations in the putative msaC ORF (Additional file 3: Figure S3.D). We then introduced the mutated operon
( $m s a A B C R \_$fsmut $m s a C$ ) into the $m s a A B C R$ deletion mutant. We compared the phenotype of this strain to the wild type strain and to the msaABCR deletion mutant complemented with wild type $m s a A B C R$. We found that the frameshift mutant operon ( $m s a A B C R_{-}$fsmut $m s a C$ ) complemented the deletion mutant in pigmentation, protease production, biofilm development, and expression of three global regulators $\operatorname{sar} A, \operatorname{agr} A$, and $\operatorname{sig} B$ (Figure 5, Table 2). The level of complementation between the msaABCR operon and msaABCR-fsmut$m s a C$ did not show a statistically significant difference (Figure 5 and Table 2). These results suggested that the $m s a C$ gene does not encode a protein. The absence of the $\mathrm{MsaC}$ protein from all current proteomic studies in $S$. aureus further supported the conclusion that $m s a C$ produces a non-protein-coding RNA that is required for the expression of the msaABCR operon $[28,29]$.

\section{Discussion}

Mutation of the $m s a C$ gene was shown to have a pleiotropic effect on the expression of a variety of genes that are involved in virulence, biofilm development, and pigmentation [21,22]. In this study, we showed that the $m s a C$ gene is part of a four-gene operon that includes two putative RNA regulators ( $m s a C$ and $m s a R$ ). We also showed that $m s a A B C R$ interacts with three global regulators $s a r A, a g r$, and $\operatorname{sig} B$. The location of the $m s a C$ gene in an operon is a significant finding because it has allowed the identification of other genes that are functionally related, and it will facilitate the study of the mechanism of regulation by this operon.

Sequence analysis of the genes in the $m s a A B C R$ operon showed that the putative protein produced by $m s a A$ is a conserved hypothetical protein in S. aureus. 


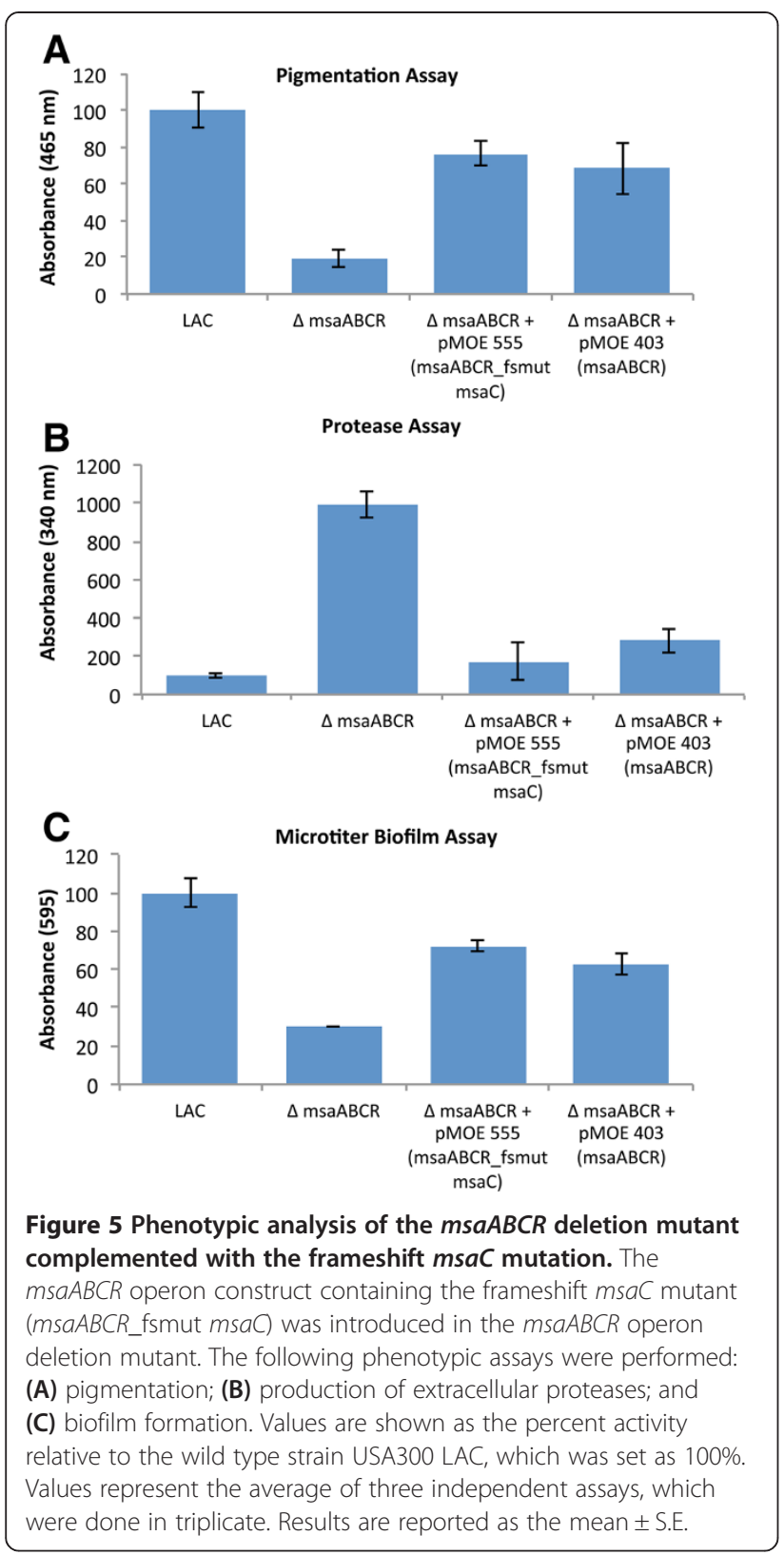

We used the I-Tasser program to predict protein structure and function of the putative MsaA protein and found that it has strong similarity to the twin-arginine signal-binding protein $[30,31]$. In many bacterial systems, twin-arginine transport is used for translocation of folded proteins across the cytoplasmic membrane [32]. In $S$. aureus, however, no such system has been described in detail. Analysis of the predicted MsaA structure also revealed that it is likely located in the cytoplasm and that it might be involved in the regulation of small GTPase-mediated signal transduction [30,31]. The contribution of the putative MsaA protein to the function of the operon remains unclear.
The $m s a B$ gene encodes a 66-amino acid polypeptide that showed homology with cold-shock proteins of E. coli (CspA, 60\%) and Bacillus subtilis (CspB, 76\%). Based on sequence homology, S. aureus produces three proteins (CspA, CspB, and CspC) that may be associated with cold-shock stress [33-35]. However, Anderson et al. [36] showed that only CspB responds to cold shock in $S$. aureus. This was confirmed by proteomic studies that showed increased expression of CspB under cold shock while CspA was not differentially expressed. Studies have also shown that the $\operatorname{csp} A$ transcript is more abundant than $\operatorname{csp} B$ and $\operatorname{csp} C$ under normal growth conditions at $37^{\circ} \mathrm{C}$, while the $\operatorname{cspB}$ transcript predominates at $15^{\circ} \mathrm{C}$ [36]. In addition, Katzif et al. [33,34] showed that $\operatorname{csp} A$ is important for the cationic antimicrobial peptide of human lysosomal cathepsin $\mathrm{G}$ and regulates pigmentation in $S$. aureus through a sigB-dependent mechanism $[33,34]$. This indicates that CspA has biological functions other than the cold-shock response. Our findings support this conclusion as we show that MsaB (CspA) acts as a regulator of several genes that are involved in protease production, virulence, and biofilm development. At this point, it is not clear how this protein interacts with other factors, but identification of the msaABCR operon will allow us to investigate this mechanism and further characterize this new regulator of virulence.

In $E$. coli, the $\operatorname{csp} A$ mRNA is a thermosensor that modulates translation of the cold-shock protein (CspA). The cspA mRNA in E. coli undergoes post-transcriptional modification in response to environmental variations such as a temperature shift from $37^{\circ} \mathrm{C}$ to $10^{\circ} \mathrm{C}[37,38]$. This RNAdependent regulation of gene expression allows $E$. coli to rapidly adapt and respond to its environment. Further studies have shown that the $\operatorname{csp} A$ gene in E. coli produces a single-stranded nucleic acid-binding protein and an RNA chaperone. This protein is one of the most abundant proteins during early growth phase, and its expression is even higher during cold shock, accounting for $2 \%$ of the total proteins in the cell [39-41]. Since MsaB (CspA) is not directly involved in cold shock in S. aureus, it is not clear if it has maintained the same mechanism of regulation or functions as its homolog in E. coli. Based on our findings and those of Lioliou et al. [25], the MsaB transcript in S. aureus binds the anti-sense RNA (msaR), which contributes to its expression. $m s a R$ is detectable in the early and midexponential growth phases but not in the late exponential phase. The $m s a B$ transcript however is still present in late exponential growth phase albeit at a slightly lower level. It is not known at this point how this correlates with the production of the MsaB protein and what the significance of the differential expression of $m s a R$ is. It is also not known if the decrease in $m s a R$ in late exponential growth phase is due to increased degradation of the anti-sense RNA or lack of production of the transcript. Lioliou et al. [25] have 
Table 2 Relative expression of global regulators in msaABCR deletion mutant complemented with the frameshift msaC mutation

\begin{tabular}{|c|c|c|c|c|}
\hline \multirow[t]{2}{*}{ Strain } & \multicolumn{4}{|c|}{ Fold change* } \\
\hline & sarA & agrA & $\operatorname{sig} B$ & $m s a C$ \\
\hline LAC & 1 & 1 & 1 & 1 \\
\hline$\triangle m s a A B C R$ & -3.59 & -6.7 & -2.07 & 0 \\
\hline$\triangle$ msaABCR + pMOE555 (msaABCR_fsmut msaC) & -1.25 & -1.04 & -1.08 & 17.95 \\
\hline$\triangle m s a A B C R+$ pMOE403 (msaABCR) & -1.39 & -1.56 & 1.01 & 16.37 \\
\hline
\end{tabular}

*Fold change in the transcription level in the various strains relative to the wild type (USA300 LAC).

Values represent the mean ratio for at least three independent experiments.

shown that anti-sense RNA ( $m s a R)$ binds to the 5' UTR region of a long transcript of $\operatorname{csp} A(m s a B)$ and prevents its processing by RNaseIII into a shorter transcript. The short transcript is presumably more stable than the long one and is translated more efficiently to produce the CspA (MsaB) protein. This processing might also be responsible to the abundance of the $m s a B$ transcript and suggests that it is the main product of the msaABCR operon. This also suggests that $\mathrm{MsaB}$ may be the main effector responsible for the functions we have shown such as biofilm development and regulation of virulence genes [21,22].

Our findings suggest that $m s a C$ produces a non-coding RNA. Our findings are supported by the absence of an $\mathrm{MsaC}$ protein from all proteomics studies in $S$. aureus. $M s a C$ RNA is expressed in the $3^{\prime}$ end of the msaB transcript and its deletion leads to a significant reduction in $m s a B$ transcript [21] and mutant phenotypes that are similar to the deletion of the whole operon msaABCR. This suggests that $m s a C$ plays a regulatory role in the expression of MsaB. Interestingly, $m s a C$ is found both as an independent transcript and part of the large operon transcript (Figure 2). We were not able to detect an $m s a C$ promoter that is active under the conditions tested, which suggested that the smaller $m s a C$ transcript was the result of processing of the large operon transcript. The mechanism by which $m s a C$ regulates the expression of $m s a B$ is not clear and requires further studies.

The identification of the msaABCR operon will add insight into the complex network of virulence regulation in S. aureus. Despite the identification of numerous regulatory elements in $S$. aureus, it is still not clear how this organism achieves the coordinated expression of virulence factors in the host. Additionally, the straindependent differences observed in the pattern of regulation of virulence in $S$. aureus complicate this problem further [3]. Therefore, the addition of the msaABCR operon to the known repertoire of regulators used by $S$. aureus and studying its interactions with other regulators will improve our understanding of staphylococcal biology and the infectious process. This study was performed using a representative strain of the USA300 clonal lineage, whose hallmark phenotype is the high production of toxins, proteases, and phenol-soluble modulins $[42,43]$. It has been suggested that the unique regulation pattern of toxins in these stains is primarily responsible for their increased virulence and epidemic spread. The msaABCR operon positively regulates the agr operon and therefore may play an important role in the phenotype of this epidemic lineage of $S$. aureus. We plan to examine the contribution of the msaABCR operon to the fine-tuning of virulence expression via $a g r$ and other regulators.

\section{Conclusions}

In this study, we identified a new operon, msaABCR, which regulates virulence and biofilm development in $S$. aureus. Two RNAs, $m s a C$ and $m s a R$, regulate expression of this operon. The $m s a C$ gene was shown to be essential for the expression and function of the operon since its deletion resulted in a similar phenotype to deletion of the whole operon. Our findings indicated that the main transcript produced by the operon was $m s a B$, which encodes the effector protein. We conclude that the pleiotropic effects observed by deletion of the $m s a A B C R$ operon are probably mediated by its interactions with the global regulators $s a r A$, agr, and $\operatorname{sig} B$. Studies are underway to define the mechanism of regulation of the $m s a A B C R$ operon and how it interacts with its target genes.

\section{Methods}

\section{Bacterial strains and plasmids}

Staphylococcus aureus strains (community-acquired MRSA strain USA300_LAC, restriction-deficient laboratory strain RN4220) and E. coli strain DH5 $\alpha$ were used in this study. $S$. aureus strains were grown in tryptic soy broth (TSB) medium. Antibiotics (chloramphenicol $(10 \mu \mathrm{g} / \mathrm{ml})$, erythromycin $(10 \mu \mathrm{g} / \mathrm{ml})$, and kanamycin $(50 \mu \mathrm{g} / \mathrm{ml}))$ were used in TSB or TSA where needed. Similarly, E. coli strains were grown in LB broth with ampicillin $(100 \mu \mathrm{g} / \mathrm{ml})$ added where needed. Detailed information about the strains and plasmid constructs used in this study is listed in supplemental Additional file 4: Table S1. 


\section{RNA isolation and real-time qPCR}

Total RNA for the Smarter ${ }^{\text {tw }}$ rapid amplification of cDNA ends (RACE) reaction was isolated from cells using a Qiagen RNeasy Maxi column (Qiagen, Valencia CA), as previously described in Sambanthamoorthy et al. [21]. Briefly, overnight cultures of S. aureus were diluted to an $\mathrm{OD}_{600}$ of 0.05 in TSB and incubated at $37^{\circ} \mathrm{C}$ with shaking $(200 \mathrm{rpm})$ until they reached an $\mathrm{OD}_{600}$ of 1.5 . The quality of total RNA was determined by Nanodrop spectrometer readings, as well as using a Bioanalyzer (Agilent). For real-time quantitative PCR (RT-qPCR) of the transcript, the total RNA was isolated from three different growth phases (early exponential, mid-exponential, and late-exponential), and RT-qPCR was performed as described previously [21]. The constitutively expressed gyrase $A$ ( $g y r A)$ gene was used as an endogenous control gene and was included in all experiments. Analysis of expression of each gene was done based on at least three independent experiments. Twofold or higher changes in gene expression were considered significant. All the primers used for RT-qPCR are listed in supplemental Additional file 5: Table S2.

\section{Analysis of RNA transcript by RACE}

Analysis of RNA transcripts was carried out using the SMARTER $^{\mathrm{Tm}}$ RACE cDNA Amplification Kit as instructed in the user manual. The locations and sequences of genespecific primers used for $3^{\prime}$ and $5^{\prime}$ RACE are shown in Figure 2 and Additional file 5: Table S2.

The 5' RACE cDNA amplification was carried out using the random primer mix $(\mathrm{N}-15)$ provided in the kit. Alternatively, for confirmatory purposes, the 5' RACE cDNA amplification was also performed using the poly (A)-tailed total RNA after poly (A) polymerization of the total RNA. The 5'-RACE-Ready cDNA was diluted to $100 \mu \mathrm{l}$ and stored at $-20^{\circ} \mathrm{C}$ until use. RACE was performed using universal primer mix, 5' RACE primers, and the Advantage 2 Polymerase mix. Control experiments and all of the optimizations for the RACE reactions were performed as instructed in the manual. RACE-amplified product $(5 \mu \mathrm{l})$ was resolved in a $1.2 \%$ gel to visualize the bands. The RACE products were gel purified and sequenced. The resulting sequence was used in a BLAST search on the NCBI website, and the $5^{\prime}$ end of the mRNA sequence was determined.

For the 3' RACE reaction, the poly (A) tail was first added to the total RNA using the Poly (A) Polymerase kit. The 3'-RACE cDNA amplification was performed with 3'SMART CDS Primer A provided in the kit. The $3^{\prime}$-RACE-Ready cDNA was diluted up to $100 \mu \mathrm{l}$ and stored at $-20^{\circ} \mathrm{C}$ until use. RACE was performed using universal primer mix, 3' RACE primers, and the Advantage 2 Polymerase mix. As above, control experiments and optimizations were performed, and the RACE products were visualized, gel purified, and sequenced. The resulting sequence was used in a BLAST search to determine the 3' end of the mRNA sequence.

\section{Northern blot analysis}

Total RNA for Northern blotting was harvested as described above. Cells were harvested at optical densities $\left(A_{600}\right)$ of $0.7,1.5$, and 4.0, which correspond to earlyexponential, mid-exponential, and late-exponential, growth phases, respectively. Northern blots were performed using the DIG Northern starter kit, according to the manufacturer's instructions (Roche Biochemicals, Mannheim, Germany). DIG-labeled riboprobes [200-300 bp] for $m s a A$, $m s a B, m s a C$, and $m s a R$ were generated by transcription using the kit. The blotted membrane was prehybridized in $25 \mathrm{ml}$ of Dig-Easy-Hyb buffer for $2 \mathrm{~h}$ at $50^{\circ} \mathrm{C}$ with rotation and hybridized in the same Dig-Easy-Hyb buffer containing $25 \mathrm{ng} / \mathrm{ml}$ DIG-labeled riboprobes overnight at $42^{\circ} \mathrm{C}$. The hybridized membrane was first washed twice with $2 \times$ SSC and $0.1 \%$ SDS for $30 \mathrm{~min}$ at $37^{\circ} \mathrm{C}$, followed by two $0.5 \times$ SSC and $0.1 \%$ SDS washes for $30 \mathrm{~min}$ at $50^{\circ} \mathrm{C}$ with rotation. After washing with $1 \times$ wash buffer (Roche) for $5 \mathrm{~min}$, the membrane was incubated with blocking solution for $60 \mathrm{~min}$ and antibody solution (anti-DIG-alkaline phosphatase, $75 \mathrm{mU} / \mathrm{ml}$ ) for $60 \mathrm{~min}$ at $37^{\circ} \mathrm{C}$ with rotation. The membrane was then equilibrated with $100 \mathrm{ml}$ of detection buffer for 2-5 min and covered with $1 \mathrm{ml}$ of the chemiluminescent substrate CDP-Star (Roche) for $10 \mathrm{~min}$ at room temperature, according to the manufacturer's protocol. The membrane was immediately exposed to film for 3-30 min.

\section{Construction of promoter-LuXAB fusions and luciferase assays}

The E. coli-staphylococcal shuttle vector pCN58, which contains the low-copy-number staphylococcal replicon cassette (pT181copwt repC) and a promoterless reporter gene, lux $A B$ (encoding the luciferase from Vibrio ficheri) for transcriptional fusions [44], was used for the study of promoter activity of the individual ORFs ( $m s a A$, $m s a B$, $m s a C$, and msaR). The upstream 200-300-bp regions from individual genes were PCR amplified and cloned into the pCN58 vector. The recombinant vectors were first transformed into RN4220, followed by transduction into the USA300 LAC strains. To study the promoterluciferase activity, overnight bacterial cultures were diluted 1:10 in TSB and further incubated for $3 \mathrm{~h}$. Cells were then normalized to OD 0.05 and further incubated at $37^{\circ} \mathrm{C}$ with shaking $(220 \mathrm{rpm})$. Bacterial cells $(5 \mathrm{ml})$ were harvested at different optical densities $\left(\mathrm{OD}_{600}\right.$ of $0.7,1.5$, and 4.0) representing early-exponential, midand late-exponential stages, respectively. The cells were washed once with $1 \times$ PBS and resuspended in $500 \mu \mathrm{l}$ of $1 \times$ PBS. The cell suspension $(500 \mu \mathrm{l})$ was mixed with $100 \mu \mathrm{l}$ of $1 \%$ decanal (v/v) in $90 \%$ ethanol, and 
luminescence was measured immediately after mixing using a luminometer, based on a 10-s measurement in the integrated data mode. Luciferase activity was recorded as relative luminescence units (RLUs), and the specific luciferase activities were calculated by dividing the RLU values by the absorbance of the organism (RLU/OD 600 ). The promoter-less version of the reporter gene plasmid (pCN58) was used as a control in reporter gene assays.

\section{Deletion of the msaABCR operon in the USA300 strain LAC and complementation}

We used a previously described mutagenesis protocol to construct a nonpolar, in-frame deletion of the $m s a A B C R$ operon in the $S$. aureus USA300 strain LAC [45]. Briefly, the flanking regions $(\sim 1 \mathrm{~kb})$ of the $m s a A B C R$ operon were amplified by PCR and ligated together at an introduced BamHI restriction site. This PCR product was inserted into the temperature-sensitive plasmid pKOR1 using the Gateway BP Clonase Enzyme Mix (Invitrogen Inc.). The pKOR1-msa operon deletion construct was introduced into $S$. aureus USA300 strain LAC. The culture was grown in TSB in the presence of chloramphenicol $(10 \mu \mathrm{g} / \mathrm{ml})$ at the permissive temperature of $30^{\circ} \mathrm{C}$. Cells were plated on TSA containing chloramphenicol at $43^{\circ} \mathrm{C}$, a non-permissive temperature for pKOR1 replication. Colonies were picked and allowed to grow in TSB at the permissive temperature and then plated on TSA containing $100 \mathrm{ng} / \mathrm{ml}$ of anhydrotetracycline, which induces antisense $\sec Y$ RNA expression and promotes loss of plasmid. Two rounds of temperature shifts were necessary to isolate the deletion mutant. Deletion of $m s a A B C R$ in LAC was verified by end-point and real-time PCR, and functional assays were performed as described previously [21]. To complement the msaABCR deletion mutation, a 1788-bp fragment of the msaABCR operon gene with complete $5^{\prime}$ and $3^{\prime}$ untranslated regions was amplified and ligated to pCN34 (NARSA), a low-copynumber, Gram-positive shuttle vector. The complement plasmid construct was introduced into strain RN4220 by electrophoresis and then transduced into the $m s a A B C R$ deletion mutant. The msaABCR operon gene in the complemented strain was under the control of its native promoter. We sequenced the agr operon of the msaABCR mutant and compared it with the parent strain to ensure that it had not spontaneously mutated during construction of the msaABCR operon deletion mutant.

\section{Mutagenesis study}

We used an overlap extension PCR cloning technique to generate a frame shift mutation in the $m s a C$ ORF as described in Bryksin et al. [46]. The upper 1120-bp fragment of the msaABCR operon was PCR amplified using primer set fsmut-msa F1 and fsmut-msa R1, and the lower 650-bp fragment of the msaABCR operon region was amplified using primer set fsmut-msa F2 and fsmut- msa R2. Primers fsmut-msa R1 and fsmut-msa F2 overlap such that a deletion of one nucleotide was introduced causing a frameshift mutation in $m s a C$. Both of the PCR fragments were PCR purified using the Promega DNA cleanup kit, and then $50 \mathrm{ng}$ of each of the fragments were used in the PCR ligation, which contained all of the components of the PCR mix except the terminal primers. The normal PCR cycle was carried out for 15 cycles, then the terminal primers (fsmut-msa F1 and fsmut-msa R2) were added to the reaction and an 20 additional cycles were performed. The final amplified PCR product was ligated to the pCN34 low copy number plasmid vector and transduced into the msaABCR operon deletion mutant.

\section{Pigmentation assay}

A pigmentation assay was performed on cells harvested from overnight cultures, as described by Morikawa et al. [47]. Briefly, $1 \mathrm{ml}$ of the cells were harvested and washed twice with water. They were then suspended in $1 \mathrm{ml}$ of methanol and heated at $55^{\circ} \mathrm{C}$ for $3-5$ min with occasional vortexing. The cells were removed by centrifugation at $15,000 \times g$ for $1 \mathrm{~min}$, and the absorbance of the supernatant was measured at $465 \mathrm{~nm}$ with water as a blank. Mean values from a minimum of three independent experiments, each performed in triplicate, were recorded.

\section{Protease assay}

Protease activity was measured from the supernatants of 4-h and overnight cultures as described by Sambanthamoorthy et al. [21]. Briefly, $300 \mu \mathrm{l}$ of the culture supernatant was mixed with $800 \mu \mathrm{l}$ of $3 \mathrm{mg}$ azocasein $\mathrm{ml}^{-1}$ in Tris-buffered saline ( $\left.\mathrm{pH} 7.5\right)$ and incubated overnight at $37^{\circ} \mathrm{C}$. Undegraded azocasein was precipitated by adding $400 \mu \mathrm{l}$ of $50 \%(\mathrm{w} / \mathrm{v})$ trichloroacetic acid, removed by centrifugation and the amount of acid-soluble azocasein was determined by measuring the $A_{340}$. Mean values from a minimum of three independent experiments, each performed in triplicate, were recorded.

\section{Biofilm assays}

The microtiter biofilm assay was performed as described in Sambanthamoorthy et al. [22] with slight modification. In brief, overnight cultures of cells, including wild type, mutant, and the complemented strain of USA300 LAC were diluted 1:100 times in TSB supplemented with $3 \% \mathrm{NaCl}$ and $0.25 \%$ glucose and inoculated in microtiter plates precoated with $20 \%$ human plasma. Cultures were incubated for 24 or $48 \mathrm{~h}$ with shaking at $150 \mathrm{rpm}$. The adherent biofilm was quantitated at $615 \mathrm{~nm}$ after washing and staining with crystal violet and elution with 5\% acetic acid. Mean values from a minimum of three independent experiments, each performed in triplicate, were recorded. 


\section{Ethics statement}

This research did not involve human subjects, human material, or human data.

\section{Additional files}

Additional file 1: Figure S1. Absolute quantification of individual ORFs in the msaABCR operon. Real-time quantitative PCR was used to compare the expression level of three genes in the msaABCR operon in three growth phases (early exponential, mid exponential, and late exponential). These results confirmed the findings from Northern blot analysis showing that despite co-expression of all genes, the msaB transcript was the most abundant. Results were obtained from three independent experiments. Values represented the mean \pm S.E.

Additional file 2: Figure S2. Activity of the ms $A B C R$ promoter. Activity of the primary promoter was measured in the msaC and msaABCR deletion mutants. Luciferase activity was measured at three planktonic growth phases (early, mid, and late exponential) and biofilm growth. The vector pCN58, containing luXAB without a promoter, was used as a negative control (not shown). Results represent the means of three independent experiments, where each measurement was done in triplicate. Values represent the mean \pm S.E.

Additional file 3: Figure S3. Predicted structure of msaC RNA and the putative protein sequence. A frame shift mutation (deletion of $U$ ) was introduced into the msaC gene of the ms $A A B C R$ operon. The predicted structure of the msaC RNA in the wild type (A) and mutant (B) strain showed no significant difference in secondary structure. The predicted protein sequence of the wild type (C) and mutant (D) strain showed the introduction of several stop codons.

Additional file 4: Table S1. Strains and plasmids used in this study. Additional file 5: Table S2. Primers used in this study.

\section{Abbreviations}

ORF: Open reading frame; RACE: Rapid amplification of cDNA ends; RLU: Relative luminescence unit; RT-qPCR: Real-time quantitative PCR.

\section{Competing interests}

The authors declare to have no competing interests.

\section{Authors' contributions}

GSS and MOE designed and performed the experiments. GSS and MOE wrote the manuscript. All authors read and approved the final manuscript.

\section{Acknowledgments}

We gratefully acknowledge the valuable technical help of Mounir Saleh, Jordan Towne, and Bina L. Jayana with this work. We are also grateful to Lindsey Shaw for sharing bacterial strain USA300 LAC and Dr. Taeok Bae for providing plasmid pKOR1. This work was funded by National Institute of Allergy and Infectious Diseases (NIAID/NIH) grant \# 1R15AI099922 (to M.O.E.) and by the Mississippi INBRE, an Institutional Development Award from the National Institute of General Medical Sciences under grant \# P20GM103476.

Received: 17 January 2014 Accepted: 5 June 2014

Published: 11 June 2014

\section{References}

1. Novick RP: Pathogenicity factors and their Regulation. In Gram-positive pathogens. Edited by Fischetti VA, Novick RP, Ferretti JJ, Portney DA, Rood I. Washinton, D C: American Society for Microbiology; 2000:392-407.

2. Tenover FC, Gaynes RP: The Epidemiology of Staphylococcus infections. In Gram positive pathogens. Edited by Fischetti VA, Novick RP, Ferrete JJ, Portney DA, Rood JI. Washintong, D C: American Society of Microbiology; 2000:414-421.

3. Junecko JM, Zielinska AK, Mrak LN, Ryan DC, Graham JW, Smeltzer M, Lee CY: Transcribing virulence in Staphylococcus aureus. World J Clin Infect Dis 2012, 2(4):63-76.
4. Felden B, Vandenesch F, Bouloc P, Romby P: The Staphylococcus aureus RNome and its commitment to virulence. PLoS Pathog 2011, 7(3):e1002006

5. Shaw LN, Lindholm C, Prajsnar TK, Miller HK, Brown MC, Golonka E, Stewart GC, Tarkowski A, Potempa J: Identification and characterization of sigma, a novel component of the Staphylococcus aureus stress and virulence responses. PLoS One 2008, 3(12):e3844.

6. Kuroda M, Ohta T, Uchiyama I, Baba T, Yuzawa H, Kobayashi I, Cui L, Oguchi A, Aoki K, Nagai Y, Lian J, Ito T, Kanamori M, Matsumaru H, Maruyama A, Murakami H, Hosoyama A, Mizutani-Ui Y, Takahashi NK, Sawano T, Inoue R, Kaito C, Sekimizu K, Hirakawa H, Kuhara S, Goto S, Yabuzaki J, Kanehisa M, Yamashita A, Oshima K, et al: Whole genome sequencing of meticillinresistant Staphylococcus aureus. Lancet 2001, 357(9264):1225-1240.

7. Peng HL, Novick RP, Kreiswirth B, Kornblum J, Schlievert P: Cloning, characterization, and sequencing of an accessory gene regulator (agr) in Staphylococcus aureus. J Bacteriol 1988, 170(9):4365-4372.

8. Cheung AL, Koomey JM, Butler CA, Projan SJ, Fischetti VA: Regulation of exoprotein expression in Staphylococcus aureus by a locus (sar) distinct from agr. Proc Natl Acad Sci U S A 1992, 89(14):6462-6466.

9. Cheung AL, Nishina KA, Trotonda MP, Tamber S: The SarA protein family of Staphylococcus aureus. Int J Biochem Cell Biol 2008, 40(3):355-361.

10. Giraudo AT, Raspanti CG, Calzolari A, Nagel R: Characterization of a Tn551mutant of Staphylococcus aureus defective in the production of several exoproteins. Can J Microbiol 1994, 40(8):677-681.

11. Fournier B, Hooper DC: A new two-component regulatory system involved in adhesion, autolysis, and extracellular proteolytic activity of Staphylococcus aureus. J Bacteriol 2000, 182(14):3955-3964.

12. Brunskill EW, Bayles KW: Identification and molecular characterization of a putative regulatory locus that affects autolysis in Staphylococcus aureus. J Bacteriol 1996, 178(3):611-618

13. Yarwood JM, McCormick JK, Schlievert PM: Identification of a novel twocomponent regulatory system that acts in global regulation of virulence factors of Staphylococcus aureus. J Bacterio/ 2001, 183(4):1113-1123.

14. Torres VJ, Stauff DL, Pishchany G, Bezbradica JS, Gordy LE, Iturregui J, Anderson KL, Dunman PM, Joyce S, Skaar EP: A Staphylococcus aureus regulatory system that responds to host heme and modulates virulence. Cell Host Microbe 2007, 1(2):109-119.

15. Gardete S, Wu SW, Gill S, Tomasz A: Role of vraSR in antibiotic resistance and antibiotic-induced stress response in Staphylococcus aureus. Antimicrob Agents Chemother 2006, 50(10):3424-3434.

16. Falord M, Mader U, Hiron A, Debarbouille M, Msadek T: Investigation of the Staphylococcus aureus GraSR regulon reveals novel links to virulence, stress response and cell wall signal transduction pathways. PLoS One 2011, 6(7):e21323.

17. Rigoulay C, Entenza JM, Halpern D, Widmer E, Moreillon P, Poquet I, Gruss A: Comparative analysis of the roles of HtrA-like surface proteases in two virulent Staphylococcus aureus strains. Infect Immun 2005, 73(1):563-572.

18. Seidl K, Stucki M, Ruegg M, Goerke C, Wolz C, Harris L, Berger-Bachi B, Bischoff M: Staphylococcus aureus $\mathrm{CcpA}$ affects virulence determinant production and antibiotic resistance. Antimicrob Agents Chemother 2006, 50(4):1183-1194.

19. Moskovitz J, Singh VK, Requena J, Wilkinson BJ, Jayaswal RK, Stadtman ER: Purification and characterization of methionine sulfoxide reductases from mouse and Staphylococcus aureus and their substrate stereospecificity. Biochem Biophys Res Commun 2002, 290(1):62-65.

20. Mei JM, Nourbakhsh F, Ford CW, Holden DW: Identification of Staphylococcus aureus virulence genes in a murine model of bacteraemia using signature-tagged mutagenesis. Mol Microbiol 1997, 26(2):399-407.

21. Sambanthamoorthy K, Smeltzer MS, Elasri MO: Identification and characterization of msa (SA1233), a gene involved in expression of sarA and several virulence factors in Staphylococcus aureus. Microbiol 2006, 152(Pt 9):2559-2572

22. Sambanthamoorthy K, Schwartz A, Nagarajan V, Elasri MO: The Role of msa in Staphylococcus aureus Biofilm Formation. BMC Microbiol 2008, 8:221.

23. Beenken KE, Blevins JS, Smeltzer MS: Mutation of sarA in Staphylococcus aureus limits biofilm formation. Infect Immun 2003, 71(7):4206-4211.

24. Nagarajan V, Elasri MO: SAMMD: Staphylococcus aureus microarray meta-database. BMC Genomics 2007, 8:351.

25. Lioliou E, Sharma CM, Caldelari I, Helfer AC, Fechter P, Vandenesch F, Vogel J, Romby P: Global regulatory functions of the Staphylococcus aureus endoribonuclease III in gene expression. PLoS Genet 2012, 8(6):e1002782. 
26. de Jong $A$, Pietersma $H$, Cordes $M$, Kuipers OP, Kok J: PePPER: a webserver for prediction of prokaryote promoter elements and regulons. $B M C$ Genomics 2012, 13:299.

27. Munch R, Hiller K, Grote A, Scheer M, Klein J, Schobert M, Jahn D: Virtual Footprint and PRODORIC: an integrative framework for regulon prediction in prokaryotes. Bioinformatics 2005, 21(22):4187-4189.

28. Cordwell SJ, Larsen MR, Cole RT, Walsh BJ: Comparative proteomics of Staphylococcus aureus and the response of methicillin-resistant and methicillin-sensitive strains to Triton X-100. Microbiol 2002, 148(Pt 9):2765-2781.

29. Ning DL, Liu CC, Liu JW, Shen Z, Chen S, Liu F, Wang BC, Yang CP: Labelfree quantitative proteomics analysis of dormant terminal buds of poplar. Mol Biol Rep 2013, 40(7):4529-4542.

30. Roy A, Kucukural A, Zhang Y: I-TASSER: a unified platform for automated protein structure and function prediction. Nat Protoc 2010, 5(4):725-738.

31. Zhang $Y$ : I-TASSER server for protein 3D structure prediction. BMC Bioinformatics 2008, 9:40.

32. Biswas L, Biswas R, Nerz C, Ohlsen K, Schlag M, Schafer T, Lamkemeyer T, Ziebandt AK, Hantke K, Rosenstein R, Gotz F: Role of the twin-arginine translocation pathway in Staphylococcus. J Bacteriol 2009, 191(19):5921-5929.

33. Katzif S, Danavall D, Bowers S, Balthazar JT, Shafer WM: The major cold shock gene, $\operatorname{csp} A$, is involved in the susceptibility of Staphylococcus aureus to an antimicrobial peptide of human cathepsin G. Infect Immun 2003, 71(8):4304-4312.

34. Katzif $S$, Lee EH, Law AB, Tzeng YL, Shafer WM: CspA regulates pigment production in Staphylococcus aureus through a SigB-dependent mechanism. J Bacteriol 2005, 187(23):8181-8184.

35. Chanda PK, Bandhu A, Jana B, Mondal R, Ganguly T, Sau K, Lee CY, Chakrabarti G, Sau S: Characterization of an unusual cold shock protein from Staphylococcus aureus. J Basic Microbiol 2010, 50(6):519-526.

36. Anderson KL, Roberts C, Disz T, Vonstein V, Hwang K, Overbeek R, Olson PD, Projan SJ, Dunman PM: Characterization of the Staphylococcus aureus heat shock, cold shock, stringent, and SOS responses and their effects on log-phase mRNA turnover. J Bacterio/ 2006, 188(19):6739-6756.

37. Brandi A, Pietroni P, Gualerzi CO, Pon CL: Post-transcriptional regulation of CspA expression in Escherichia coli. Mol Microbiol 1996, 19(2):231-240.

38. Giuliodori AM, Di Pietro F, Marzi S, Masquida B, Wagner R, Romby P, Gualerzi CO, Pon CL: The cspA mRNA is a thermosensor that modulates translation of the cold-shock protein CspA. Mol Cell 2010, 37(1):21-33.

39. Goldstein J, Pollitt NS, Inouye M: Major cold shock protein of Escherichia coli. Proc Natl Acad Sci U S A 1990, 87(1):283-287.

40. La Teana A, Brandi A, Falconi M, Spurio R, Pon CL, Gualerzi CO: Identification of a cold shock transcriptional enhancer of the Escherichia coli gene encoding nucleoid protein H-NS. Proc Natl Acad Sci U S A 1991, 88(23):10907-10911.

41. Jiang $W$, Hou $Y$, Inouye M: CspA, the major cold-shock protein of Escherichia coli, is an RNA chaperone. J Biol Chem 1997, 272(1):196-202.

42. Tenover FC, Goering RV: Methicillin-resistant Staphylococcus aureus strain USA300: origin and epidemiology. J Antimicrob Chemother 2009, 64(3):441-446

43. Joo HS, Cheung GY, Otto M: Antimicrobial activity of communityassociated methicillin-resistant Staphylococcus aureus is caused by phenol-soluble modulin derivatives. J Biol Chem 2011, 286(11):8933-8940.

44. Charpentier E, Anton Al, Barry P, Alfonso B, Fang Y, Novick RP: Nove cassette-based shuttle vector system for gram-positive bacteria. Appl Environ Microbiol 2004, 70(10):6076-6085.

45. Bae T, Schneewind O: Allelic replacement in Staphylococcus aureus with inducible counter-selection. Plasmid 2006, 55(1):58-63.

46. Bryksin AV, Matsumura I: Overlap extension PCR cloning: a simple and reliable way to create recombinant plasmids. Biotechniques 2010 48(6):463-465.

47. Morikawa K, Maruyama A, Inose Y, Higashide M, Hayashi H, Ohta T: Overexpression of sigma factor, sigma(B), urges Staphylococcus aureus to thicken the cell wall and to resist beta-lactams. Biochem Biophys Res Commun 2001, 288(2):385-389.

doi:10.1186/1471-2180-14-154

Cite this article as: Sahukhal and Elasri: Identification and

characterization of an operon, $m s a A B C R$, that controls virulence and biofilm development in Staphylococcus aureus. BMC Microbiology 2014 14:154

\section{Submit your next manuscript to BioMed Central and take full advantage of:}

- Convenient online submission

- Thorough peer review

- No space constraints or color figure charges

- Immediate publication on acceptance

- Inclusion in PubMed, CAS, Scopus and Google Scholar

- Research which is freely available for redistribution
C Biomed Central 